approach. The overall findings, suggest reductions in medical mistrust and anti-LGBT stigma.

Disclosure No significant relationships.

\section{S21.3 AUTHENTIC AND SUCCESSFUL COMMUNITY ENGAGEMENT FOR STI/HIV PREVENTION, SCREENING AND TREATMENT IN RURAL AND URBAN SETTINGS}

Scott Rhodes*. Wake Forest School of Medicine, Social Science and Health Policy, WinstonSalem, USA

10.1136/sextrans-2019-sti.95

Sexually transmitted infections (STIs) and HIV remain critical public health challenges in the United States. There is a clear need for innovative approaches that identify, prioritize, and address underlying 'upstream' social determinants of health while developing and harnessing community assets to improve STI and HIV prevention, screening, and treatment. Community engagement has emerged as an approach to improve public health outcomes, including reduced STIs and HIV. However, community engagement is difficult, and this presentation provides guidance on the characteristics and strategies of authentic and successful community engagement designed to promote STI and HIV prevention, screening, and treatment in both rural and urban settings. Members of our community partnerships used mixed-methods to identify characteristics of successful community engagement and associated strategies across more than 30 STI and HIV initiatives across the United States. We abstracted data from existing project documentation including proposal documents, project-specific logic models, team and partnership meeting notes, and other materials. Partnership members examined these documents and used an iterative approach with review, discussion, and re-review. A component of our analysis was to identify characteristics and strategies that crossed initiatives, had potential to be generalizable, and could guide future STI and HIV initiatives. Some of the characteristics and strategies that were identified included, staff/team knowledge and unflagging commitment to community engagement; understanding of and commitment to social justice; structural flexibility; strong and charismatic institutional leadership; participation of partners representing diverse multiple sectors; recognizing, acknowledging, and reducing power differentials; shared decision making; embracing and working through conflict; identifying and leveraging talent, strengths, and resources; and using a stepwise approach to build a shared history. Often and incorrectly conflated with advisory boards, qualitative methods, and behavioral and social sciences more broadly, community engagement requires careful forethought, the use of inclusive processes that bring together diverse constituencies, and shared resources and power.

Disclosure No significant relationships.

\section{S21.4 SPURRING INNOVATION IN IMPROVING COMMUNITY ENGAGEMENT FOR STI PREVENTION}

Weiming Tang *. UNC Project-China, Guangzhou, China

10.1136/sextrans-2019-sti.96

Improving the input from the community is an approach for STI Prevention. For example, by engaging the contribution of the community, a 'pay-it-forward' dramatically improved chlamydia and gonorrhea testing among Chinese men who have sex with men (MSM). In this presentation, we summarized how innovative tools were used to engage different groups of the population for STI prevention. First, crowdsourcing approaches were used to solicit the inputs from the community for developing new intervention approaches, designing intervention messages, and evaluating intervention programs. Crowdsourcing involves a group of non-experts and experts working together to solve a problem and then sharing solutions with the public. Second, designathon and hackathon methods were adjusted to develop STI intervention messages and platforms among key populations. For example, in China, a 3-day designathon (hackathon-like event) was hosted to develop an HIV testing service strategy for MSM, while MSM were invited to join the event. In 2019, a hackathon event was also conducted in China to develop a platform for MSM to seek for gay-friendly health service. Third, social-marketing and other participatory approaches were used to develop intervention tools for improving STI services.

Disclosure No significant relationships.

\section{Clinical Case Series}

\section{CCSO1 - CLINICAL CASE SERIES - SYPHILIS: THE GREAT IMITATOR}

\section{Monday, July 15, 2019 7:00 AM - 8:00 AM}

\section{CCSO1.1 NEUROSYPHILIS IS MORE COMMON AMONG MALIGNANT SYPHILIS: BASED ON CASE SERIES EVIDENCE}

Pingyu Zhou* ${ }^{*}$ Lin Zhu. Shanghai Skin Disease Hospital, Shanghai, China

\subsection{6/sextrans-2019-sti.97}

Background Malignant syphilis and neurosyphilis were believed more likely to exist in the HIV positive population. However China's huge and active epidemics of syphilis were much more seen in HIV negative population, thus a considerable amount of the malignant syphilis patients with HIV-uninfected patients might be under the shadow of lower evaluate of neurosyphilis.

Methods This study aimed to investigate the relationship between HIV infection, malignant syphilis and neurosyphilis through a systematic analysis.

Results Clinical characteristics of the malignant syphilis: 26 patients were diagnosed with malignant syphilis and eighteen out of them were HIV negative. They presented different symptoms, and had a more aggressive disease progression with skin rashes. Laboratory findings: 1) A strongly high titre of serum RPR and a sub-acute inflammation of the histopathological finds; The RPR titers of all patients were strongly positive with a range from 1:32 to 1:256. Histopathological examination demonstrated a sub-acute inflammation rich with dermal perivascular plasma cell and neutrophils infiltrate. Numerous spirochetes will be found by immunohistochemistry. 2) WBC, protein and VDRL index in CSF. CSF test revealed the increased WBC count $(>10$ cells $/ \mu \mathrm{L})$ in eleven patients, of which five were with HIV positive. Increased total protein $(>0.5 \mathrm{~g} / \mathrm{L})$ were found in eight patients, and four of them 